\title{
Surfen auf den langen Wellen?
}

\author{
Sogenannte Schlüsselinnovationen sind in zweierlei Hinsicht interessant. Sie \\ gelten zum einen als Wachstumsstimulans, denn von ihnen wird eine Erneve- \\ rung der kapitalistischen Wachstumsdynamik erwartet. Zum anderen verändern \\ Innovationen die Beschaffenheit des industriellen Metabolismus. Insofern kann \\ man fragen, ob diese Innovationen in die richtige ökologische Richtung gehen. \\ Doch trotz aller berechtigten Kritik an Innovationen und ihren Wirkungen gilt, \\ dass sie bislang unverzichtbar sind.
}

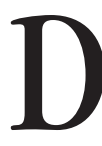
ass sich der technische Fortschritt erschöpfen kann, ist eine recht alte Finsich der Ökonomie. Schon 1912 schrieb Wolf: ,Wir haben derart ein Gesetz der technisch-ökonomischen Entwicklungsgrenze, welches besagt, dass jeder die Produktionskosten ermäßigende oder die Nutzung von Stoff und Kraft vervollkommende technische Fortschritt dem weiteren Fortschritt den Weg verlegt, ihm immer weniger zu tun übrig lässt, welche Einengung der Fortschrittsmöglichkeiten dann aber notwendig zur Folge hat, dass der technische Fortschritt, weil immer weniger lohnend, mindestens in gewisser Richtung erlahmt und schließlich, weil unergiebig, für ganze Gebiete nicht mehr gesucht wird“ (1). Modern formuliert: die Verfolgung einer technisch-ökonomischen Trajektorie führt zu einem abnehmenden Grenzertrag der Forschungsaufwendungen. Der abnehmende Grenzertrag von FuE-Aufwendungen ist eine wichtige Triebfeder den technischen Fortschritt immer wieder zu erneuern, oder, um im Sprachgebrauch von Wolf zu bleiben, für den technischen Fortschritt neue Gebiete zu suchen. Daraus erklärt sich zu einem guten Teil das Interesse an Schlïsselinnovationen.

Was man sich unter Schlüsselinnovationen vorstellen soll, ist allerdings in der Literatur nicht eindeutig geklärt. Zwei Charakteristika lassen sich aber vielleicht dennoch aus dem allgemeinen Sprachgebrauch herausdestillieren. Schlüsselinnovationen repräsentieren erstens eine spezielle Art von technischen Erneuerungen, die fundamentale Neuerungen tradierter technischer Problemlösungen repräsentieren - hier gibt es eine enge Verwandtschaft zum Begriff der Basisinnovation. Zweitens charakterisiert Schlüsselinnovationen die Erwartung von einer sehr großen Anwendungsbreite- sie sind nicht nur eine Neuerung in einem speziellen, sondern in vielen ver-
Diese Diskussion ist weitgehend wieder vergessen. Die politische Schlussfolgerung hat sich jedoch gehalten und ist mittlerweile in allen politischen Lagern ubiquitär. Eine erlahmende Wachstumsdynamik des Kapitalismus könne durch Basis- oder eben Schlüsselinnovationen neu befeuert werden Der Staat täte demzufolge gut daran, durch eine intelligente Technologiepolitik, seinen Anteil zu Schlüsselinnovationen beitragen.

\section{- Kein Beweis für Wachstum durch technologischen Wandel}

Auch wenn das Argumentationsmuster die Plausibilität auf seiner Seite hat, ist sein theoretisches Fundament durchaus angreifbar. So wurde immer wieder bestritten, dass sich die von Kondratieff postulierten langen Wellen überhaupt empirisch nachweisen lassen. Ihre statistische Unterfütterung hatte immer mit der Schwierigkeit zu kämpfen, dass in den langen Zeitreihen stets methodische Brüche der Erhebung der volkswirtschaftlichen Aggregatgrößen stecken. Auch der Zusammenhang zur Innovationstätigkeit steht auf einer schwachen Basis, weil der empirische Nachweis einer stetigen Erfindungstätigkeit und einer zyklischen Einführung von technischen Neuerungen in die wirtschaftliche Praxis, wie sie etwa Mensch im Sinne hatte, methodisch auch alles andere als einfach ist.

Außerdem kann man natürlich kritisch fragen, ob der Staat überhaupt über die Steuerungskompetenz verfügt, solche Wellen zu glätten, wenn er sich schon bei der Steuerung wesentlich kurzfristiger laufender Variablen offensichtlich schwer tut. Da viele technische Neuerungen kaum ohne staatliche Mithilfe denkbar sind, soll hier nicht einem technologischen Nachtwächterstaat das Wort geredet werden. Die zunehmende Verquickung von staatlicher Technologieförderung und privater Innovationstätigkeit ist ein säkulärer Trend, der sich kaum rückgängig machen lässt. Die Frage, ob der staatliche Akteur allerdings die Erfolg versprechenden zukünftigen Träger langer Wellen wird identifizieren können und ob diese wirklich für eine beschleunigte Umsetzung von Technik in wirtschaftlichen Erfolg sorgen können, ist aber durchaus berechtigt. Die vielen Investitionsruinen aus 30 Jahren Technologieförderung belegen, dass staatliche Akteure es eben auch oft nicht besser wissen als private und sich vielfach von Scientific Communities oder Unternehmensverbünden zu umfangreichen Subventionen verleiten lassen, die sich hernach als Fehlinvestition entpuppen. 
Die Technologiepolitik in Europa hat es bis heute nicht vermocht, die Wachstumsdynamik auch nur annähernd in die Nähe jener Wachstumsraten zu bringen, die charakteristisch für die 50er Jahre waren. Die zwischenzeitlich rege Wachstumsdynamik in den USA oder Japan lässt sich auch ganz gut anders als mit dem technischen Fortschritt erklären, etwa mit einer großzügigeren Geldpolitik der amerikanischen Notenbank oder einer Stimulierung durch keynesianische Nachfrageprogramme etwa im Rüstungsbereich. Ob das Versprechen von Wohlfahrt durch technologieinduzierte Steigerung der Wachstumsdynamik wirklich eingelöst werden kann, darf also bezweifelt werden.

\section{Schlüsselinnovationen und ökolo- gische Balance}

Kritiker des technischen Fortschritts haben sich immer mit der Frage auseinandersetzt, ob der technische Fortschritt die menschliche Gattung aus einem Gleichgewicht mit der Natur reißt. Dieses Motiv ist sehr alt und lässt sich von der Prometheus-Sage über Malthus bis Rachel Carson verfolgen (4). Wenngleich heute das Bekenntnis wohlfeil ist, dass es keinen Weg zurück zu einem wie auch immer imaginierten prästabilen Urzustand menschlicher Gesellschaften gibt, ist diese Idee in neuer sprachlicher Verkleidung noch immer virulent. Ein Fundstück dieser Art repräsentieren die Überlegungen von Paech, der formuliert: „,Die Suche nach dem Neuen, noch nicht vorhandenen, soll Probleme lösen, deren Ursachen jedoch - gerade bei Nachhaltigkeitsproblemen - alles andere als nicht vorhanden oder etwa unbekannt sind. Ein schlichter Vergleich verdeutlicht das Fatale an dieser Logik: Anstatt die Folgen eines eingenommenen Giftes ursachenadäquat zu beheben, also das Gift abzusetzen, wird ein Gegengift verabreicht“ (5). Zugespitzt interpretiert: die unerwünschten Folgen der technischer Neuerungen lassen sich nicht mit technischen Neuerungen kurieren.

Seine Idee, nämlich aus dem Innovationsroulette auszusteigen, führt jedoch vermutlich nicht weit. Auch wenn man mit guten Argumenten der Meinung ist, dass nicht jede Technologie auch angewendet werden sollte, nur weil sie neu ist, gibt es keinen Ausstieg aus der Technosphäre. Sie gleicht wohl eher einem Boot, das auf hoher See fortwährend umgebaut wird. Das Boot kann nicht verlassen werden und man kann das Boot auch nicht einfach so lassen wie es ist, denn jede Form von Technik ist Lösung alter und Quelle neuer Probleme zugleich. In den Begriffen der Systemtheorie könnte man auch sagen, menschliche Gesell- schaften befinden sich in einem instabilen Gleichgewicht fernab von jedem natürlichen Gleichgewicht. Da dieses Gleichgewicht instabil ist, muss es immer wieder - auch durch technischen Fortschritt - erneuert werden.

Es gibt in dieser Hinsicht zum technischen Fortschritt und damit zu Innovationen keine reale ökologische Alternative. Auch Ökologen müssen sich wohl mit der Idee anfreunden, dass jede Veränderung der Technosphäre, auch die, die unter Suffizienzvorzeichen gedacht wird, eine Bewegung in nur teilweise bekanntem Fahrwasser ist und immer wieder Risiken und Probleme heraufbeschwört, die erst nachdem sie manifest geworden sind, gesellschaftlich bearbeitet werden können. Die gilt auch für Schlüsseltechnologien. Sie können trotz der hohen Erwartungen nie ein Allheilmittel sein, werden vermutlich neue ökologische Probleme aufwerfen und sind aber zur Lösung bestehender Probleme unverzichtbar. Gegen unsere prinzipielle Wissensbeschränkungen, was uns alles in Folge des technischen Fortschritts widerfahren könnte, ist noch kein Kraut gewachsen.

\section{Metaphysik der Zulässigkeit not- wendig}

Allerdings sind solche prinzipiellen Überlegungen kein ökologischer Freifahrtschein für jegliche Art von technischem Fortschritt. Einige Vorsichtsmaßnahmen auf der Fahrt in unbekannte Gewässer lassen sich durchaus treffen. Technologiefolgenabschätzung und die ökobilanzielle Bewertung von möglichen Entwicklungsszenarien erlauben Einsichten in künftige Möglichkeiten und Risiken. Entwicklungsmoratorien sind Mittel, erst einmal Erfahrungen zu sammeln und vor allem zu reflektieren, bevor ein großer technologischer Sprung gewagt wird. Schließlich tut auch jede moderne Gesellschaft gut daran, sich nicht in eine völlige Abhängigkeit von spezifischen Technologien zu begeben. Technologische Alternativen sollten schon verfügbar sein, wenn sich eine Technologie im Zuge ihrer Verbreitung als problematisch entpuppt. Eine gewisse Redundanz technologischer Systeme macht Sinn.

Die spannende Frage, inwieweit sich ex ante Technologiebewertungen mit Hilfe von verallgemeinerbaren Regeln vornehmen lassen, ist aber noch keineswegs abschließend beantwortet. Von Gleich hat vor etlichen Jahren das Kriterium der Eingriffstiefe vorgeschlagen, mit dessen Hilfe genetische oder Manipulationen auf atomarer Ebene diskriminiert werden sollten. Offensichtlich ist die historische Entwicklung über diese Idee hinwegge-

\begin{tabular}{|c|}
\hline Einen Überblick über alle bisher \\
erschienen Hefte des \\
Informationsdienstes \\
"Ökologisches Wirtschaften" \\
finden Sie auf unseren Internetseiten \\
unter der Adresse: \\
w w w. o e k o m. de \\
Wir freuen uns auf Ihren Besuch!
\end{tabular}

gangen, aber man darf durchaus fragen, ob ohne eine Metaphysik der Zulässigkeit - Metaphysik, weil sie eben nicht vollständig mit naturwissenschaftlicher Erkenntnis gedeckt werden kann - der technische Fortschritt in Zukunft sinnvoll weitergehen kann. Vielleicht demonstriert uns die Gentechnologie, dass man den Hebel für eine solche Metaphysik weniger in den möglichen äußeren ökologischen Grenzen von technischen Neuerungen, sondern vielmehr in unserem Menschenbild und in unserer Kultur suchen sollte. Es könnte ja sein, dass uns die Schlüsseltechnologie Gentechnik so schön wie Brad Pitt, so erfolgreich wie Bill Gates, so mächtig wie George Bush und obendrein unsterblich macht. Aber wollen wir das wirklich?

\section{Anmerkungen}

(1) Wolf zitiert nach Berg, Christoph: Technologischer Fortschritt und ökonomische Regulierung: ein evolutionärer Ansatz mit einer Fallstudie zur Entstehung und Entwicklung der Rübenzuckerindustrie in Deutschland. Frankfurt a.M. 1995, S. 45.

(2) Programmatisch dafür steht die folgende Monographie: Hauff, V./Scharpf, F.: Modernisierung der Volkswirtschaft - Technologiepolitik als Strukturpolitik. Frankfurt a.M./Köln 1975.

(3) Mensch, G.: Innovation und industrielle Evolution. International Institute of Management. Berlin 1973.

(4) wer sich für die Klassiker des ökologischen Denkens interessiert, sei auf folgenden Sammelband verwiesen: Nellisen, N./ van der Straaten, J./Klinker, L.: Classics in Enviromental Studies - An Overview of Classic Texts in Enviromental Studies. Utrecht 1997

(5) Paech, N.: Nebenwirkungen nicht ausgeschlossen Innovationen und Nachhaltigkeit. In: Politische Ökologie: Innovationen, Heft 84, 2003, S. 17.

\section{Die Autor}

Dr. Stefan Zundel ist Professor für Umweltökonomie und Volkswirtschaftslehre an der FH Lausitz. Kontakt: Fachbereich Wirtschaftswissenschaften, FH Lausitz, Großenhainer Straße 57, 01968 Senftenberg. Tel. 03573-85733, E-Mail: zundel@fh-lausitz.de 
(c) 20I0 Authors; licensee IÖW and oekom verlag. This is an article distributed under the terms of the Creative Commons Attribution Non-Commercial No Derivates License (http://creativecommons.org/licenses/by-nc-nd/3.o/), which permits unrestricted use, distribution, and reproduction in any medium, provided the original work is properly cited. 